\title{
The Existence of Positive Solution to a Nonlinear Fractional Differential Equation with Integral Boundary Conditions
}

\author{
Meiqiang Feng, ${ }^{1}$ Xiaofang Liu, ${ }^{1}$ and Hanying Feng ${ }^{2}$ \\ ${ }^{1}$ School of Applied Science, Beijing Information Science \& Technology University, Beijing, 100192, China \\ ${ }^{2}$ Department of Mathematics, Shijiazhuang Mechanical Engineering College, Shijiazhuang 050003, China
}

Correspondence should be addressed to Meiqiang Feng, meiqiangfeng@sina.com

Received 19 December 2010; Accepted 26 January 2011

Academic Editor: J. J. Trujillo

Copyright (C) 2011 Meiqiang Feng et al. This is an open access article distributed under the Creative Commons Attribution License, which permits unrestricted use, distribution, and reproduction in any medium, provided the original work is properly cited.

The expression and properties of Green's function for a class of nonlinear fractional differential equations with integral boundary conditions are studied and employed to obtain some results on the existence of positive solutions by using fixed point theorem in cones. The proofs are based upon the reduction of problem considered to the equivalent Fredholm integral equation of second kind. The results significantly extend and improve many known results even for integer-order cases.

\section{Introduction}

Fractional calculus is an area having a long history, its infancy dates back to three hundred years, the beginnings of classical calculus. It had attracted the interest of many old famous mathematicians, such as L'Hospital, Leibniz, Liouville, Riemann, Grünward, Letnikov, and so forth $[1,2]$. As the old mathematicians expected, in recent several decades fractional differential equations have been found to be a powerful tool in more and more fields, such as materials, physics, mechanics, and engineering [1-5]. For the basic theory and recent development of the subject, we refer the reader to a text by Lakshmikantham et al. [6]. For more details and examples, see [7-24] and the references therein. However, the theory of boundary value problems for nonlinear fractional differential equations is still in the initial stages, and many aspects of this theory need to be explored. In [13], Bai and Lü used the fixed point theorems to show the existence and multiplicity of positive solutions to the nonlinear fractional boundary value problem

$$
\begin{gathered}
\mathrm{D}^{\alpha} u(t)+f(t, u(t))=0, \quad 0<t<1, \\
u(0)=0, \quad u(1)=0,
\end{gathered}
$$


where $1<\alpha \leq 2, \mathrm{D}^{\alpha}$ is the standard Riemann-Liouville differentiation, and $f:[0,1] \times[0, \infty) \rightarrow$ $[0, \infty)$ is a given continuous function.

In [15], Zhang showed the existence and multiplicity of positive solutions of the fractional boundary value problem

$$
\begin{gathered}
\mathrm{D}_{0+}^{\alpha} u(t)=f(t, u(t)), \quad 0<t<1, \\
u(0)+u^{\prime}(0)=0, \quad u(1)+u^{\prime}(1)=0,
\end{gathered}
$$

where $1<\alpha \leq 2$ is a real number, and $\mathbf{D}_{0+}^{\alpha}$ is the Caputo's fractional derivative. The function $f$ is continuous on $[0,1] \times[0, \infty)$.

Recently, Ahmad and Nieto [11] investigated some existence results for a nonlinear fractional integrodifferential equation with integral boundary conditions

$$
\begin{gathered}
{ }^{c} \mathbf{D}^{q} x(t)+f(t, x(t),(x x)(t))=0, \quad 0<t<1,1<q \leq 2, \\
\alpha x(0)+\beta x^{\prime}(0)=\int_{0}^{1} q_{1}(x(s)) d s, \\
\alpha x(1)+\beta x^{\prime}(1)=\int_{0}^{1} q_{2}(x(s)) d s,
\end{gathered}
$$

where ${ }^{c} \mathbf{D}^{q}$ is the Caputo fractional derivative, $f:[0,1] \times X \times X \rightarrow X$, for $\curlyvee:[0,1] \times[0,1] \rightarrow$ $[0, \infty)$,

$$
(x x)=\int_{0}^{1} \curlyvee(t, s) x(s)
$$

$q_{1}, q_{2}: X \rightarrow X, \alpha>0, \beta \geq 0$ are real numbers, and $X$ is a Banach space.

Being directly inspired by $[11,13,15]$, we intend in this paper to study the following boundary value problems of fractional order differential equation

$$
\begin{aligned}
& \mathbf{D}_{0+}^{\alpha} x(t)+g(t) f(t, x)=0, \quad 0<t<1, \\
& x(0)=0, \quad x^{\prime}(1)=\int_{0}^{1} h(t) x(t) d t
\end{aligned}
$$

where $1<\alpha \leq 2, g \in C((0,1),[0,+\infty))$ and $g$ may be singular at $t=0$ or $/$ and at $t=1, \mathbf{D}_{0+}^{\alpha}$ is the standard Riemann-Liouville differentiation, $h \in L^{1}[0,1]$ is nonnegative, and $f \in C([0,1] \times$ $[0,+\infty),[0,+\infty))$.

In the case of $h(t) \equiv 0$, for all $t \in[0,1]$, boundary value problem (1.5) reduces to the problem studied by Kaufmann and Mboumi [19]. In [19], the authors used the fixed point theorems to show sufficient conditions for the existence of at least one and at least three positive solutions to problem (1.5). For the case of $\alpha=2$, boundary value problem (1.5) is related to a boundary value problems of integer-order differential equation. Feng et al. [25] considered the existence and multiplicity of positive solutions to boundary value problem (1.5) by applying the fixed point theory in a cone for strict set contraction operators. 
The organization of this paper is as follows. We will introduce some lemmas and notations in the rest of this section. In Section 2, we present the expression and properties of Green's function associated with boundary value problem (1.5). In Section 3, we give some preliminaries about operator. In particular, we state fixed point theory in cones. In Section 4, the main results of boundary value problem (1.5) will be stated and proved. In Section 5, we offer some interesting discussion of the associated boundary value problem (1.5). Finally, conclusions in Section 6 close the paper.

The fractional differential equations-related notations adopted in this paper can be found, if not explained specifically, in almost all literature related to fractional differential equations. The readers who are unfamiliar with this area can consult for example [1-6] for details.

Definition 1.1 (see [4]). The integral

$$
I_{0+}^{\alpha} f(x)=\frac{1}{\Gamma(\alpha)} \int_{0}^{x} \frac{f(t)}{(x-t)^{1-\alpha}} d t, \quad x>0
$$

where $\alpha>0$ is called Riemann-Liouville fractional integral of order $\alpha$.

Definition 1.2 (see [4]). For a function $f(x)$ given in the interval $[0,1)$, the expression

$$
D_{0+}^{\alpha} f(x)=\frac{1}{\Gamma(n-\alpha)}\left(\frac{d}{d x}\right)^{n} \int_{0}^{x} \frac{f(t)}{(x-t)^{\alpha-n+1}} d t
$$

where $n=[\alpha]+1,[\alpha]$ denotes the integer part of number $\alpha$, is called the Riemann-Liouville fractional derivative of order $\alpha$.

Lemma 1.3 (see [13]). Assume that $u \in C(0,1) \cap L(0,1)$ with a fractional derivative of order $\alpha>0$ that belongs to $u \in C(0,1) \cap L(0,1)$. Then

$$
I_{0+}^{\alpha} D_{0+}^{\alpha} u(t)=u(t)+C_{1} t^{\alpha-1}+C_{2} t^{\alpha-2}+\cdots+C_{N} t^{\alpha-N},
$$

for some $C_{i} \in R, i=1,2, \ldots, N$, where $N$ is the smallest integer greater than or equal to $\alpha$.

\section{Expression and Properties of Green's Function}

In this section, we present the expression and properties of Green's function associated with boundary value problem (1.5).

Lemma 2.1. Assume that $\int_{0}^{1} h(t) t^{\alpha-1} d t \neq \alpha-1$. Then for any $y \in C[0,1]$, the unique solution of boundary value problem

$$
\begin{gathered}
\mathbf{D}_{0+}^{\alpha} x(t)+y(t)=0, \quad 0<t<1, \\
x(0)=0, \quad x^{\prime}(1)=\int_{0}^{1} h(t) x(t) d t,
\end{gathered}
$$


is given by

$$
x(t)=\int_{0}^{1} G(t, s) y(s) d s,
$$

where

$$
\begin{gathered}
G(t, s)=G_{1}(t, s)+G_{2}(t, s), \\
G_{1}(t, s)= \begin{cases}\frac{t^{\alpha-1}(1-s)^{\alpha-2}-(t-s)^{\alpha-1}}{\Gamma(\alpha)}, & 0 \leq s \leq t \leq 1, \\
\frac{t^{\alpha-1}(1-s)^{\alpha-2}}{\Gamma(\alpha)}, & 0 \leq t \leq s \leq 1,\end{cases} \\
G_{2}(t, s)=\frac{t^{\alpha-1}}{\alpha-1-\int_{0}^{1} h(t) t^{\alpha-1} d t} \int_{0}^{1} h(t) G_{1}(t, s) d t .
\end{gathered}
$$

Proof. By Lemma 1.3, we can reduce the equation of problem (2.1) to an equivalent integral equation

$$
x(t)=-I_{0+}^{\alpha} y(t)+c_{1} t^{\alpha-1}+c_{2} t^{\alpha-2}=-\frac{1}{\Gamma(\alpha)} \int_{0}^{t}(t-s)^{\alpha-1} y(s) d s+c_{1} t^{\alpha-1}+c_{2} t^{\alpha-2} .
$$

By $x(0)=0$, there is $c_{2}=0$, and

$$
x^{\prime}(t)=-\frac{\alpha-1}{\Gamma(\alpha)} \int_{0}^{t}(t-s)^{\alpha-2} y(s) d s+c_{1}(\alpha-1) t^{\alpha-2} .
$$

By (2.7) and $x^{\prime}(1)=\int_{0}^{1} h(t) x(t) d t$, we have

$$
\int_{0}^{1} h(t) x(t) d t=-\frac{\alpha-1}{\Gamma(\alpha)} \int_{0}^{1}(1-s)^{\alpha-2} y(s) d s+c_{1}(\alpha-1),
$$

which yields that

$$
c_{1}=\frac{1}{\alpha-1} \int_{0}^{1} h(t) x(t) d t+\frac{1}{\Gamma(\alpha)} \int_{0}^{1}(1-s)^{\alpha-2} y(s) d s
$$


Therefore, the unique solution of BVP (2.1) is

$$
\begin{aligned}
x(t) & =-\frac{1}{\Gamma(\alpha)} \int_{0}^{t}(t-s)^{\alpha-1} y(s) d s+t^{\alpha-1}\left\{\frac{1}{\alpha-1} \int_{0}^{1} h(t) x(t) d t+\frac{1}{\Gamma(\alpha)} \int_{0}^{1}(1-s)^{\alpha-2} y(s) d s\right\} \\
& =\int_{0}^{1} G_{1}(t, s) y(s) d s+\frac{t^{\alpha-1}}{\alpha-1} \int_{0}^{1} h(t) x(t) d t,
\end{aligned}
$$

where $G_{1}(t, s)$ is defined by $(2.4)$.

Multiplying (2.10) with $h(t)$ and integrating it, we can see

$$
\int_{0}^{1} h(t) x(t) d t=\int_{0}^{1} h(t) \int_{0}^{1} G_{1}(t, s) y(s) d s d t+\frac{\int_{0}^{1} h(t) t^{\alpha-1} d t}{\alpha-1} \int_{0}^{1} h(t) x(t) d t
$$

Therefore,

$$
\int_{0}^{1} h(t) x(t) d t=\frac{1}{1-\int_{0}^{1} h(t) t^{\alpha-1} d t /(\alpha-1)} \int_{0}^{1} h(t) \int_{0}^{1} G_{1}(t, s) y(s) d s d t .
$$

Substituting (2.12) into (2.10), we obtain

$$
\begin{aligned}
x(t) & =\int_{0}^{1} G_{1}(t, s) y(s) d s+\frac{t^{\alpha-1}}{\alpha-1} \int_{0}^{1} h(t) x(t) d t \\
& =\int_{0}^{1} G_{1}(t, s) y(s) d s+\frac{t^{\alpha-1}}{\alpha-1} \frac{1}{1-\int_{0}^{1} h(t) t^{\alpha-1} d t /(\alpha-1)} \int_{0}^{1} h(t) \int_{0}^{1} G_{1}(t, s) y(s) d s d t \\
& =\int_{0}^{1} G_{1}(t, s) y(s) d s+\int_{0}^{1} G_{2}(t, s) y(s) d s \\
& =\int_{0}^{1} G(t, s) y(s) d s,
\end{aligned}
$$

where $G(t, s), G_{1}(t, s)$, and $G_{2}(t, s)$ are defined by (2.3), (2.4), and (2.5), respectively. The proof is complete.

From (2.3), (2.4), and (2.5), we can prove that $G(t, s), G_{1}(t, s)$, and $G_{2}(t, s)$ have the following properties. 
Proposition 2.2. The function $G_{1}(t, s)$ defined by (2.4) satisfies the following.

(i) $G_{1}(t, s) \geq 0$ is continuous for all $t, s \in[0,1], G_{1}(t, s)>0$, for all $t, s \in(0,1)$;

(ii) $G_{1}(t, s) \leq G_{1}(s, s)=s^{\alpha-1}(1-s)^{\alpha-2} / \Gamma(\alpha)$, for all $t \in[0,1], s \in(0,1)$.

Proof. (i) It is obvious that $G_{1}(t, s)$ is continuous on $[0,1] \times[0,1]$. For $0 \leq s<t \leq 1$,

$$
t^{\alpha-1}(1-s)^{\alpha-2}-(t-s)^{\alpha-1}=(1-s)^{\alpha-1}\left[\frac{t^{\alpha-1}}{1-s}-\left(\frac{t-s}{1-s}\right)^{\alpha-1}\right] \geq 0
$$

So, by (2.4), we have

$$
G_{1}(t, s) \geq 0, \quad \forall t, s \in[0,1] .
$$

Similarly, for $t, s \in(0,1)$, we have $G_{1}(t, s)>0$.

(ii) Since $\alpha \leq 2$, for given $s \in(0,1), s<t \leq 1$, we have

$$
\begin{gathered}
t \geq \frac{t-s}{1-s} \\
t^{\alpha-2} \leq\left(\frac{t-s}{1-s}\right)^{\alpha-2} .
\end{gathered}
$$

Therefore, from (2.17) and the definition of $G_{1}(t, s)$, for given $s \in(0,1), s<t \leq 1$, we have

$$
\begin{aligned}
\frac{\partial G_{1}(t, s)}{\partial t} & =\frac{\alpha-1}{\Gamma(\alpha)}\left\{t^{\alpha-2}(1-s)^{\alpha-2}-(t-s)^{\alpha-2}\right\} \\
& =\frac{\alpha-1}{\Gamma(\alpha)}(1-s)^{\alpha-2}\left\{t^{\alpha-2}-\left(\frac{t-s}{1-s}\right)^{\alpha-2}\right\} \\
& \leq 0
\end{aligned}
$$

On the other hand, it is clear that

$$
\frac{\partial G_{1}(t, s)}{\partial t}=\frac{(\alpha-1) t^{\alpha-2}(1-s)^{\alpha-2}}{\Gamma(\alpha)} \geq 0, \quad \forall 0 \leq t \leq s \leq 1 .
$$

Therefore, we have

$$
\max _{t \in[0,1]} G_{1}(t, s)=G_{1}(s, s)=\frac{s^{\alpha-1}(1-s)^{\alpha-2}}{\Gamma(\alpha)}, \quad s \in(0,1) .
$$

Let

$$
\mu=\int_{0}^{1} h(t) t^{\alpha-1} d t
$$


Proposition 2.3. If $\mu \in[0, \alpha-1)$, then one has

(i) $G_{2}(t, s) \geq 0$ is continuous for all $t, s \in[0,1], G_{2}(t, s)>0$, for all $t, s \in(0,1)$;

(ii) $G_{2}(t, s) \leq(1 /(\alpha-1-\mu)) \int_{0}^{1} h(t) G_{1}(t, s) d t$, for all $t \in[0,1], s \in(0,1)$.

Proof. From the properties of $G_{1}(t, s)$, and the definition of $G_{2}(t, s)$, we can prove that the results of Proposition 2.3 hold.

Theorem 2.4. If $\mu \in[0, \alpha-1)$, the function $G(t, s)$ defined by (2.3) satisfies the following.

(i) $G(t, s) \geq 0$ is continuous for all $t, s \in[0,1], G(t, s)>0$, for all $t, s \in(0,1)$;

(ii) $G(t, s) \leq \Lambda s^{\alpha-1}(1-s)^{\alpha-2}$, for all $t, s \in[0,1]$, where

$$
\Lambda=\frac{\alpha-1-\mu-\int_{0}^{1} h(t) d t}{\Gamma(\alpha)(\alpha-1-\mu)} .
$$

Proof. (i) From Propositions 2.2 and 2.3, we obtain that $G(t, s) \geq 0$ is continuous for all $t, s \in$ $[0,1], G(t, s)>0$, for all $t, s \in(0,1)$.

(ii) From Proposition 2.2 and (2.3), we have

$$
\begin{aligned}
G(t, s) & =G_{1}(t, s)+G_{2}(t, s) \\
& \leq G_{1}(s, s)+\frac{t^{\alpha-1}}{\alpha-1-\mu} \int_{0}^{1} h(t) G_{1}(s, s) d t \\
& \leq G_{1}(s, s)\left(1+\frac{1}{\alpha-1-\mu} \int_{0}^{1} h(t) d t\right) \\
& \leq G_{1}(s, s) \frac{\alpha-1-\mu+\int_{0}^{1} h(t) d t}{\alpha-1-\mu} \\
& \leq \frac{s^{\alpha-1}(1-s)^{\alpha-2}}{\Gamma(\alpha)} \frac{\alpha-1-\mu-\int_{0}^{1} h(t) d t}{\alpha-1-\mu} \\
& =\Lambda s^{\alpha-1}(1-s)^{\alpha-2}, \quad \forall t, s \in[0,1] .
\end{aligned}
$$

Remark 2.5. From (i) of Theorem 2.4, we obtain that there exists $\tau>0$ such that

$$
G(t, s) \geq \tau, \quad \forall t, s \in[\theta, 1-\theta]
$$

where $\theta \in(0,1 / 2)$.

\section{Preliminaries}

In this section, we give some preliminaries for discussing the existence of positive solutions of boundary value problem (1.5). 
Let $J=[0,1]$. The basic space used in this paper is $E=C[0,1]$. It is well known that $E$ is a real Banach space with the norm $\|\cdot\|$ defined by $\|x\|=\max _{0 \leq t \leq 1}|x(t)|$. Let

$$
\begin{gathered}
K=\{x \in E: x(t) \geq 0, t \in J\}, \\
K_{r}=\{x \in K:\|x\| \leq r\}, \quad \partial K_{r}=\{x \in K:\|x\|=r\},
\end{gathered}
$$

where $r>0$.

On the basis of Lemma 3.3 below we will establish in Section 4 the existence of positive solution to the problem (1.5). Here we make the following hypotheses:

$\left(H_{1}\right) g \in C((0,1),[0,+\infty)), g(t) \not \equiv 0$ on any subinterval of $(0,1)$ and $\int_{0}^{1} s^{\alpha-1}(1-s)^{\alpha-2} g(t) d t<$ $+\infty$;

$\left(H_{2}\right) f \in C([0,1] \times[0,+\infty),[0,+\infty))$ and $f(t, 0)=0$ uniformly with respect to $t$ on $[0,1]$;

$\left(H_{3}\right) \mu \in[0, \alpha-1)$, where $\mu$ is defined by $(2.21)$.

Define $T: K \rightarrow K$ by

$$
(T x)(t)=\int_{0}^{1} G(t, s) g(s) f(s, x(s)) d s,
$$

where $G(t, s)$ is defined by $(2.3)$.

Lemma 3.1. Let $\left(\mathrm{H}_{1}\right)-\left(\mathrm{H}_{3}\right)$ hold. Then boundary value problems (1.5) has a solution $x$ if and only if $x$ is a fixed point of $T$.

Proof. From Lemma 2.1, we can prove the results of this Lemma.

Lemma 3.2. Let $\left(H_{1}\right)-\left(H_{3}\right)$ hold. Then $T K \subset K$ and $T: K \rightarrow K$ is completely continuous.

Proof. For any $x \in K$, by (3.2), we can obtain $T x \geq 0$. Next by similar proof of Lemma 3.1 in [12] and Ascoli-Arzela theorem one can prove $T: K \rightarrow K$ is completely continuous. So it is omitted.

Lemma 3.3 (see [26]). Let $\Omega_{1}$ and $\Omega_{2}$ be two bounded open sets in a real Banach space $E$, such that $0 \in \Omega_{1}$ and $\bar{\Omega}_{1} \subset \Omega_{2}$. Let operator $A: P \cap\left(\bar{\Omega}_{2} \backslash \Omega_{1}\right) \rightarrow P$ be completely continuous, where $P$ is a cone in E. Suppose that one of the following two conditions is satisfied.

(i) There exists $x_{0} \in P \backslash\{\theta\}$ such that $x-A x \neq t x_{0}$, for all $x \in P \cap \partial \Omega_{2}, t \geq 0 ; A x \neq \mu x$, for all $x \in P \cap \partial \Omega_{1}, \mu \geq 1$.

(ii) There exists $x_{0} \in P \backslash\{\theta\}$ such that $x-A x \neq t x_{0}$, for all $x \in P \cap \partial \Omega_{1}, t \geq 0 ; A x \neq \mu x$, for all $x \in P \cap \partial \Omega_{2}, \mu \geq 1$.

Then, $A$ has at least one fixed point in $P \cap\left(\Omega_{2} \backslash \bar{\Omega}_{1}\right)$.

\section{Existence of Positive Solutions}

In this section, we apply Lemma 3.3 to establish the existence of positive solutions for boundary value problems (1.5). 
Theorem 4.1. Suppose $\left(H_{1}\right)-\left(H_{3}\right)$ and $f$ satisfies the following conditions.

$\left(H_{4}\right)$ There exists $0<\delta<1$ such that $0<\lim _{\inf } \rightarrow 0^{+} \min _{t \in[0,1]}\left(f(t, x) / x^{\delta}\right) \leq+\infty$;

$\left(H_{5}\right)$ There exists $0<\beta<1$ such that $0 \leq \limsup _{x \rightarrow+\infty} \max _{t \in[0,1]}\left(f(t, x) / x^{\beta}\right)<+\infty$.

Then boundary value problems (1.5) has at least one positive solution.

Proof. For applying Lemma 3.3, we construct a function $w:[0,1] \rightarrow R$ via

$$
w(t)= \begin{cases}1, & t \in[\theta, 1-\theta], \\ 0, & t \notin\left[\frac{\theta}{16}, 1-\frac{15 \theta}{16}\right], \\ \frac{16}{15 \theta}\left(t-\frac{\theta}{16}\right), & t \in\left[\frac{\theta}{16}, \theta\right], \\ -\frac{16}{\theta}\left(t-1+\frac{15 \theta}{16}\right), & t \in\left[1-\theta, 1-\frac{15 \theta}{16}\right] .\end{cases}
$$

Obviously, $w$ is a nonnegative continuous function, that is, $w \in K$, and $\|w\|=1$.

Suppose that there is $\varepsilon_{1}>0$ such that

$$
x-T x \neq 0 \quad\left(\forall x \in K, 0<\|x\| \leq \varepsilon_{1}\right),
$$

if not, then the conclusion holds. The condition $\left(H_{4}\right)$ and $f(t, 0)=0$ imply that there exist $\sigma>0, \varepsilon_{2}>0$ such that

$$
f(t, x) \geq \sigma x^{\delta} \quad\left(0 \leq x \leq \varepsilon_{2}\right)
$$

Let $\varepsilon_{3}=\min \left\{\varepsilon_{1}, \varepsilon_{2},\left(\tau \sigma \int_{\theta}^{1-\theta} g(s) d s\right)^{1 /(1-\delta)}\right\}$, and choose $0<r<\varepsilon_{3}$. We now show that

$$
x-T x \neq \zeta w \quad\left(\forall x \in \partial K_{r}, \zeta \geq 0\right) .
$$

In fact, if there exist $x_{1} \in \partial K_{r}, \zeta_{1} \geq 0$ such that $x_{1}-T x_{1}=\zeta_{1} w$, then (4.4) implies that $\zeta_{1}>0$. On the other hand, $x_{1}=\zeta_{1} w+T x_{1} \geq \zeta_{1} w$. So we can choose $\zeta^{*}=\sup \left\{\zeta \mid x_{1} \geq t w\right\}$, then $\zeta_{1} \leq \zeta^{*}<+\infty, x_{1} \geq \zeta^{*} w$. Therefore,

$$
\zeta^{*}=\zeta^{*}\|w\| \leq\left\|x_{1}\right\|=r<\varepsilon_{3} \leq\left(\tau \sigma \int_{\theta}^{1-\theta} g(s) d s\right)^{1 /(1-\delta)}
$$


Consequently, for any $t \in[\theta, 1-\theta],(2.24)$ and (4.3) imply

$$
\begin{aligned}
x_{1}(t) & =\int_{0}^{1} G(t, s) g(s) f\left(s, x_{1}(s)\right) d s+\zeta_{1} w(t) \\
& \geq \int_{0}^{1} G(t, s) g(s) \sigma\left[x_{1}(s)\right]^{\delta} d s+\zeta_{1} w(t) \\
& \geq \int_{\theta}^{1-\theta} G(t, s) g(s) \sigma\left(\zeta^{*}\right)^{\delta}[w(s)]^{\delta} d s+\zeta_{1} w(t) \\
& \geq \tau \sigma\left(\zeta^{*}\right)^{\delta} \int_{\theta}^{1-\theta} g(s) d s+\zeta_{1} w(t) \\
& \geq\left(\zeta^{*}+\zeta_{1}\right) w(t)
\end{aligned}
$$

that is, $x_{1}(t) \geq\left(\zeta^{*}+\zeta_{1}\right) w(t), t \in[\theta, 1-\theta]$. Noticing the definition of $w(t)$, we have

$$
x_{1}(t) \geq\left(\zeta^{*}+\zeta_{1}\right) w(t), \quad t \in[0,1]
$$

which is a contradiction to the definition of $\zeta^{*}$. Hence, (4.4) holds.

Now turning to $\left(H_{5}\right)$, there exist $m>0, \varepsilon_{4}>0$, for $t \in[0,1], x \geq \varepsilon_{4}$, such that $f(t, x) \leq$ $m x^{\beta}$. Letting $l=\max _{0 \leq t \leq 1,0 \leq x \leq \varepsilon_{4}} f(t, x)$, then

$$
0 \leq f(t, x) \leq m x^{\beta}+l, \quad \forall t \in[0,1], x \in[0, \infty)
$$

Choosing $R>\varepsilon_{4}$ such that

$$
\frac{l \Lambda M}{R}+\frac{m \Lambda M}{R^{1-\beta}}<1
$$

where $M=\int_{0}^{1} s^{\alpha-1}(1-s)^{\alpha-2} g(s) d s$. Now we prove that

$$
T x \neq \lambda x, \quad \forall x \in \partial K_{R}, \lambda \geq 1 \text {. }
$$

If not, then there exist $x_{0} \in \partial K_{R}, \lambda_{0} \geq 1$ such that $T x_{0}=\lambda_{0} x_{0}$. By (4.8) and (ii) of Theorem 2.4, then for any $t \in[0,1]$, we have

$$
\begin{aligned}
\lambda_{0} x_{0}(t) & =\int_{0}^{1} G(t, s) g(s) f\left(s, x_{0}(s)\right) d s \\
& \leq\left(l+m\left\|x_{0}\right\|^{\beta}\right) \Lambda \int_{0}^{1} s^{\alpha-1}(1-s)^{\alpha-2} g(s) d s .
\end{aligned}
$$


So $R \leq \lambda_{0} R=\lambda_{0}\left\|x_{0}\right\| \leq\left(l+m\left\|x_{0}\right\|^{\beta}\right) \Lambda \int_{0}^{1} s^{\alpha-1}(1-s)^{\alpha-2} g(s) d s$, that is,

$$
\frac{l \Lambda M}{R}+\frac{m \Lambda M}{R^{1-\beta}} \geq 1
$$

which is a contradiction to (4.9). So, (4.10) holds.

By (ii) of Lemma 3.3, (4.4) and (4.10) yield that $T$ has a fixed point $x \in K_{r, R}, r \leq\|x\| \leq$ $R$. Thus it follows that boundary value problems (1.5) has at least one positive solution $x$ with $r \leq\|x\| \leq R$. The proof is complete.

\section{Discussion}

In this section, we offer some interesting discussion associated with boundary value problems (1.5).

Since the proof of the main theorem (Theorem 4.1) in this paper is independent of the expression form of $G(t, s)$ and only dependent on its continuity and nonnegativity, there are similar conclusions by analogous methods for boundary value problems (1.5) subject to other boundary value conditions, respectively, the following.

(i) We have

$$
x(0)=0, \quad x^{\prime}(1)=\int_{0}^{1} h(t) x^{\prime}(t) d t,
$$

then

$$
G(t, s)=G_{1}(t, s)+G_{2}^{*}(t, s),
$$

where

$$
\begin{gathered}
G_{2}^{*}(t, s)=\frac{t^{\alpha-1}}{(\alpha-1)\left(1-\int_{0}^{1} h(t) t^{\alpha-2} d t\right)} \int_{0}^{1} h(t) G_{1 t}^{\prime}(t, s) d t, \\
G_{1 t}^{\prime}(t, s)= \begin{cases}\frac{(\alpha-1) t^{\alpha-2}(1-s)^{\alpha-2}-(\alpha-1)(t-s)^{\alpha-2}}{\Gamma(\alpha)}, & 0 \leq s \leq t \leq 1, \\
\frac{(\alpha-1) t^{\alpha-2}(1-s)^{\alpha-2}}{\Gamma(\alpha)}, & 0 \leq t \leq s \leq 1 .\end{cases}
\end{gathered}
$$

Obviously $G(t, s)$ is continuous on $[0,1] \times[0,1]$, and it is easy to see that $G(t, s) \geq$ $0, t, s \in[0,1]$ by $\mu^{*} \in[0,1)$, where

$$
\mu^{*}=\int_{0}^{1} h(t) t^{\alpha-2} d t
$$


(ii) We have

$$
x(0)=0, \quad x(1)+x^{\prime}(1)=\int_{0}^{1} h(t) x(t) d t
$$

then

$$
G(t, s)=G_{1}^{*}(t, s)+G_{2}^{* *}(t, s),
$$

where

$$
\begin{gathered}
G_{1}^{*}(t, s)= \begin{cases}\frac{t^{\alpha-1}(1-s)^{\alpha-1}+(\alpha-1)(1-s)^{\alpha-2}-\alpha(t-s)^{\alpha-1}}{\alpha \Gamma(\alpha)}, & 0 \leq s \leq t \leq 1, \\
\frac{t^{\alpha-1}(1-s)^{\alpha-1}+(\alpha-1)(1-s)^{\alpha-2}}{\alpha \Gamma(\alpha)}, & 0 \leq t \leq s \leq 1 .\end{cases} \\
G_{2}^{* *}(t, s)=\frac{t^{\alpha-1}}{\left(\alpha-\int_{0}^{1} h(t) t^{\alpha-1} d t\right)} \int_{0}^{1} h(t) G_{1}^{*}(t, s) d t .
\end{gathered}
$$

Obviously $G(t, s)$ is continuous on $[0,1] \times[0,1]$, and it is easy to see that $G(t, s) \geq 0$, $t, s \in[0,1]$ by $\mu \in[0, \alpha)$, where $\mu$ is defined by $(2.21)$.

(iii) We have

$$
x(0)=0, \quad x(1)+x^{\prime}(1)=\int_{0}^{1} h(t) x^{\prime}(t) d t
$$

then

$$
G(t, s)=G_{1}^{*}(t, s)+G_{2}^{* * *}(t, s)
$$

where

$$
\begin{gathered}
G_{2}^{* * *}(t, s)=\frac{t^{\alpha-1}}{(\alpha-1)\left(\alpha /(\alpha-1)-\int_{0}^{1} h(t) t^{\alpha-2} d t\right)} \int_{0}^{1} h(t) G_{1 t}^{*^{\prime}}(t, s) d t, \\
G_{1 t}^{* \prime}(t, s)= \begin{cases}\frac{(\alpha-1) t^{\alpha-2}(1-s)^{\alpha-1}-(\alpha-1) \alpha(t-s)^{\alpha-2}}{\alpha \Gamma(\alpha)}, & 0 \leq s \leq t \leq 1, \\
\frac{(\alpha-1) t^{\alpha-2}(1-s)^{\alpha-1}}{\alpha \Gamma(\alpha)}, & 0 \leq t \leq s \leq 1 .\end{cases}
\end{gathered}
$$

Obviously $G(t, s)$ is continuous on $[0,1] \times[0,1]$, and it is easy to see that $G(t, s) \geq$ $0, t, s \in[0,1]$ by $\mu^{*} \in[0, \alpha /(\alpha-1))$, where $\mu^{*}$ is defined in (5.4). 


\section{Conclusions}

In this paper, by using the fixed point theorem of cone, we have investigated the existence of positive solutions for a class of nonlinear fractional differential equations with integral boundary conditions and have obtained some easily verifiable sufficient criteria which extend previous results. It is worth mentioning that there are still many problems that remain open in this vital field other than the results obtained in this paper: for example, whether or not we can study the fractional differential equations with integral boundary conditions at resonance (see, e.g., [27]), and whether or not we can give a unified approach applicable to many BVPs (see, e.g., [28-31]). More efforts are still needed in the future.

\section{Acknowledgments}

The authors thank the referee for his/her careful reading of the paper and useful suggestions. This paper is sponsored by the Funding Project for Academic Human Resources Development in Institutions of Higher Learning under the jurisdiction of Beijing Municipality (PHR201008430), the Scientific Research Common Program of Beijing Municipal Commission of Education (KM201010772018), the Natural Sciences Foundation of Heibei Province (A2009001426), and the Beijing Excellent Training Grant (2010D005007000002).

\section{References}

[1] K. B. Oldham and J. Spanier, The Fractional Calculus, Academic Press, London, UK, 1974.

[2] I. Podlubny, Fractional Differential Equations, vol. 198 of Mathematics in Science and Engineering, Academic Press, San Diego, Calif, USA, 1999.

[3] K. S. Miller and B. Ross, An Introduction to the Fractional Calculus and Fractional Differential Equations, A Wiley-Interscience Publication, John Wiley \& Sons, New York, NY, USA, 1993.

[4] S. G. Samko, A. A. Kilbas, and O. I. Marichev, Fractional Integrals and Derivatives, Gordon and Breach Science Publishers, Yverdon, Switzerland, 1993.

[5] A. A. Kilbas, H. M. Srivastava, and J. J. Trujillo, Theory and Applications of Fractional Differential Equations, vol. 204 of North-Holland Mathematics Studies, Elsevier Science, Amsterdam, The Netherlands, 2006.

[6] V. Lakshmikantham, S. Leela, and J. Vasundhara Devi, Theory of Fractional Dynamic Systems, Cambridge Academic, Cambridge, UK, 2009.

[7] V. Lakshmikantham and A. S. Vatsala, "Basic theory of fractional differential equations," Nonlinear Analysis. Theory, Methods \& Applications, vol. 69, no. 8, pp. 2677-2682, 2008.

[8] V. Daftardar-Gejji, "Positive solutions of a system of non-autonomous fractional differential equations," Journal of Mathematical Analysis and Applications, vol. 302, no. 1, pp. 56-64, 2005.

[9] V. Daftardar-Gejji and S. Bhalekar, "Boundary value problems for multi-term fractional differential equations," Journal of Mathematical Analysis and Applications, vol. 345, no. 2, pp. 754-765, 2008.

[10] B. Ahmad and J. J. Nieto, "Existence and approximation of solutions for a class of nonlinear impulsive functional differential equations with anti-periodic boundary conditions," Nonlinear Analysis. Theory, Methods \& Applications, vol. 69, no. 10, pp. 3291-3298, 2008.

[11] B. Ahmad and J. J. Nieto, "Existence results for nonlinear boundary value problems of fractional integrodifferential equations with integral boundary conditions," Boundary Value Problems, vol. 2009, Article ID 708576, 11 pages, 2009.

[12] Z. Bai, "On positive solutions of a nonlocal fractional boundary value problem," Nonlinear Analysis. Theory, Methods E Applications, vol. 72, no. 2, pp. 916-924, 2010.

[13] Z. Bai and H. Lü, "Positive solutions for boundary value problem of nonlinear fractional differential equation," Journal of Mathematical Analysis and Applications, vol. 311, no. 2, pp. 495-505, 2005. 
[14] D. Jiang and C. Yuan, "The positive properties of the Green function for Dirichlet-type boundary value problems of nonlinear fractional differential equations and its application," Nonlinear Analysis. Theory, Methods E Applications, vol. 72, no. 2, pp. 710-719, 2010.

[15] S. Zhang, "Positive solutions for boundary-value problems of nonlinear fractional differential equations," Electronic Journal of Differential Equations, no. 36, pp. 1-12, 2006.

[16] M. Benchohra, S. Hamani, and S. K. Ntouyas, "Boundary value problems for differential equations with fractional order and nonlocal conditions," Nonlinear Analysis. Theory, Methods \& Applications, vol. 71, no. 7-8, pp. 2391-2396, 2009.

[17] H. A. H. Salem, “On the nonlinear Hammerstein integral equations in Banach spaces and application to the boundary value problem of fractional order," Mathematical and Computer Modelling, vol. 48, no. 7-8, pp. 1178-1190, 2008.

[18] H. A. H. Salem, “On the fractional order m-point boundary value problem in reflexive Banach spaces and weak topologies," Journal of Computational and Applied Mathematics, vol. 224, no. 2, pp. 565-572, 2009.

[19] E. R. Kaufmann and E. Mboumi, "Positive solutions of a boundary value problem for a nonlinear fractional differential equation," Electronic Journal of Qualitative Theory of Differential Equations, no. 3, pp. 1-11, 2008.

[20] C. F. Li, X. N. Luo, and Y. Zhou, "Existence of positive solutions of the boundary value problem for nonlinear fractional differential equations," Computers $\mathcal{E}$ Mathematics with Applications, vol. 59, no. 3, pp. 1363-1375, 2010.

[21] S. Zhang, "The existence of a positive solution for a nonlinear fractional differential equation," Journal of Mathematical Analysis and Applications, vol. 252, no. 2, pp. 804-812, 2000.

[22] S. Zhang, "Existence of positive solution for some class of nonlinear fractional differential equations," Journal of Mathematical Analysis and Applications, vol. 278, no. 1, pp. 136-148, 2003.

[23] W. Zhong and W. Lin, "Nonlocal and multiple-point boundary value problem for fractional differential equations," Computers \& Mathematics with Applications, vol. 59, no. 3, pp. 1345-1351, 2010.

[24] M. Feng, X. Zhang, and W. Ge, "New existence results for higher-order nonlinear fractional differential equation with integral boundary conditions," Boundary Value Problems, vol. 2011, Article ID 720702, 20 pages, 2011.

[25] M. Feng, D. Ji, and W. Ge, "Positive solutions for a class of boundary-value problem with integral boundary conditions in Banach spaces," Journal of Computational and Applied Mathematics, vol. 222, no. 2, pp. 351-363, 2008.

[26] D. Guo, V. Lakshmikantham, and X. Liu, Nonlinear Integral Equations in Abstract Spaces, vol. 373 of Mathematics and Its Applications, Kluwer Academic Publishers, Dordrecht, The Netherlands, 1996.

[27] X. Zhang, M. Feng, and W. Ge, "Existence result of second-order differential equations with integral boundary conditions at resonance," Journal of Mathematical Analysis and Applications, vol. 353, no. 1, pp. 311-319, 2009.

[28] G. Infante and J. R. L. Webb, "Nonlinear non-local boundary-value problems and perturbed Hammerstein integral equations," Proceedings of the Edinburgh Mathematical Society. Series II, vol. 49, no. 3, pp. 637-656, 2006.

[29] J. R. L. Webb, "Positive solutions of some three point boundary value problems via fixed point index theory," Nonlinear Analysis. Theory, Methods \& Applications, vol. 47, no. 7, pp. 4319-4332, 2001.

[30] J. R. L. Webb and G. Infante, "Positive solutions of nonlocal boundary value problems: a unified approach," Journal of the London Mathematical Society. Second Series, vol. 74, no. 3, pp. 673-693, 2006.

[31] J. R. L. Webb, G. Infante, and D. Franco, "Positive solutions of nonlinear fourth-order boundary-value problems with local and non-local boundary conditions," Proceedings of the Royal Society of Edinburgh. Section A, vol. 138, no. 2, pp. 427-446, 2008. 